\title{
La pharmacie hospitalière au Canada : une longueur d'avance
}

\author{
par James E Tisdale and Kevin W Hall
}

$\mathrm{E}^{\mathrm{n}}$ 2008, l'American Society of Health-System Pharmacists (ASHP) et sa Fondation pour la recherche et l'éducation ont mis de l'avant le projet de modèle d'exercice de la pharmacie (Pharmacy Practice Model Initiative ou PPMI), dont le but est "d'améliorer sensiblement la santé et le bien-être des patients dans les établissements de santé en élaborant et en disséminant des modèles optimaux d'exercice de la pharmacie basés sur l'utilisation la plus efficace des pharmaciens comme fournisseurs de soins directs aux patients $»^{1}$. Certains des principaux objectifs du PPMI pour les établissements de santé sont de décrire des modèles optimaux d'exercice de la pharmacie qui favorisent la prestation sécuritaire, efficace, efficiente et responsable des soins liés à la pharmacothérapie des patients; d'accroître la capacité des techniciens en pharmacie; de déterminer des services fondamentaux liés aux soins des patients qui devraient être fournis systématiquement par les services de pharmacie; de favoriser la compréhension et le soutien des modèles optimaux d'exercice de la pharmacie par les patients et les aidants, les professionnels de la santé, les dirigeants du domaine des soins de santé et les payeurs; de cerner les technologies présentes et futures nécessaires au soutien des modèles optimaux d'exercice de la pharmacie; et de définir des mesures précises que les pharmaciens d'hôpitaux devraient prendre pour mettre en place des modèles optimaux d'exercice de la pharmacie.

En novembre 2010, l'ASHP a tenu une conférence sur invitation, le sommet sur le modèle d'exercice de la pharmacie, à laquelle ont participé plus de 100 chefs de file de la pharmacie afin d'en arriver à un consensus sur les types de modèles d'exercice de la pharmacie qui répondraient le mieux aux besoins des patients et des établissements de santé qui assurent la prestation des soins à ces patients. Les recommandations consensuelles issues du sommet sont nombreuses, mais en voici certaines des plus essentielles ${ }^{2}$ :

- Les pharmaciens des établissements de santé doivent être responsables des résultats liés à la pharmacothérapie des patients.

- La gestion de la pharmacothérapie doit être assurée par un pharmacien pour chacun des patients hospitalisés.

- $\quad$ Pourvus des titres de compétence requis et des privilèges ainsi accordés, les pharmaciens doivent inclure dans leur champ de pratique la prescription dans le cadre de l'équipe de soins en collaboration.

- Toutes les fonctions de distribution ne nécessitant pas de jugement clinique doivent être dévolues aux techniciens en pharmacie.

- Les pharmaciens qui assurent la gestion de la pharmacothérapie doivent avoir réussi un programme de résidence agréé ou détenir une expérience équivalente.

- Les pharmaciens qui assurent la gestion de la pharmacothérapie doivent être agréés par le processus de certification le plus pertinent du Conseil des spécialités en pharmacie (Board of Pharmacy Specialties).

Le sommet sur le modèle d'exercice de la pharmacie n'a pas donné lieu à des recommandations précises quant à l'adoption d'un ou de plusieurs modèles d'exercice particuliers, mais a plutôt débouché sur des appels à " prendre des mesures audacieuses qui propulseront la pharmacie hospitalière vers l'avenir " pour les services de pharmacie de réformer l'utilisation des pharmaciens, des techniciens et des ressources technologiques $»^{4}$. Le compte rendu du sommet comportait des exemples qualifiés de "modèles d'exercice de pointe ". Un de ces exemples pour un petit hôpital communautaire était de faire participer le pharmacien à une équipe de soins de santé en collaboration composée d'un médecin, d'une infirmière ou d'un infirmier et d'un gestionnaire de soins ${ }^{5}$. Toutefois, de tels modèles sont en place dans de nombreux établissements depuis des dizaines d'années, et on serait en droit d'affirmer que le qualificatif " de pointe " à mettre la barre plutôt basse. Le modèle d'exercice " de pointe " donné en exemple pour un grand centre médical universitaire était un modèle décentralisé dans lequel " du personnel clinique ou des spécialistes " non définis étaient déployés, avec des techniciens en pharmacie décentralisés, vers de multiples unités de soins aux patients, dans le but de réduire au minimum le travail non clinique des pharmaciens grâce notamment à la plus grande utilisation de la technologie et des techniciens en pharmacie ${ }^{6}$. Bien que ces stratégies soient importantes, elles ne sont ni nouvelles, ni particulièrement innovantes — la plus 
grande utilisation des techniciens et de la technologie ayant été prônée par les chefs de file de la profession depuis le début des années $1960^{7,8}$.

Nous sommes de ceux qui croient que le PPMI aux ÉtatsUnis a été trop timide dans sa préconisation des modèles de pointe d'exercice de la pharmacie. Des développements récents au Canada nous portent à croire que l'exercice de la pharmacie au Canada occupe une place de choix pour réussir un changement transformationnel dans l'exercice de la pharmacie hospitalière qui placerait la barre plus haut que ne le fait le PPMI de l'ASHP.

D'abord, la réglementation et l'agrément des techniciens en pharmacie, déjà en vigueur dans plusieurs provinces canadiennes et qui le seront très bientôt dans la plupart des autres, créent une véritable occasion pour les techniciens en pharmacie réglementés d'assumer la responsabilité des aspects techniques de la distribution des médicaments. Bien que certains services de pharmacie continuent de croire que les pharmaciens doivent jouer un rôle majeur dans les systèmes de distribution des médicaments, beaucoup d'autres ont déjà adopté ou visent des modèles d'exercice dont le but est de mettre en place des systèmes de distribution des médicaments informatisés sous la responsabilité des techniciens, qui permettraient de libérer les pharmaciens pour qu'ils puissent se concentrer presque exclusivement sur les soins aux patients et l'optimisation des résultats thérapeutiques $<9>$. Faute de réglementation et d'agrément des techniciens en pharmacie, la responsabilité finale du pharmacien pour ce qui est des systèmes de distribution des médicaments a été perçue comme un obstacle et s'est avérée être un obstacle au dégagement du pharmacien des activités techniques de distribution des médicaments. Or, cet obstacle a commencé à s'effriter, du fait de la mise en place rapide à l'échelle du Canada de la réglementation des techniciens en pharmacie.

Le pharmacien a aussi été aux prises avec l'absence du droit de prescrire pour pouvoir gérer pleinement la pharmacothérapie, mais cet obstacle aux modèles de pointe d'exercice de la pharmacie s'effrite également. L'Alberta en a été la protagoniste en accordant le droit de prescrire autonome aux pharmaciens ${ }^{10}$ et la plupart des autres provinces sont en train d'apporter ou apporteront des changements à leur réglementation afin d'accorder aux pharmaciens le droit de prescrire à un niveau qui leur permettra d'assumer une responsabilité accrue dans la gestion de la pharmacothérapie.

L'exercice de la pharmacie hospitalière au Canada n'a certes pas mis en ouvre l'ensemble des recommandations issues du sommet sur le modèle d'exercice de la pharmacie. La capacité d'accueil des programmes de résidence est un problème sérieux au Canada et aux États-Unis et il faudra vraisemblablement un certain temps avant que tous les pharmaciens du Canada gérant la pharmacothérapie aient terminé un programme de résidence agréé. En outre, bien quau milieu de 2012 on comptait 163 pharmaciens canadiens agréés par le Board of Pharmacy Specialties (d'après la base de données sur les pharmaciens agréés du Conseil au www.bpsweb.org/resources/find_bcp.cfm), cet agrément n’a pas encore obtenu la reconnaissance générale au Canada. Les chefs de file de l'exercice de la pharmacie hospitalière au Canada devront décider s'ils accordent de la valeur aux titres de compétences et aux privilèges accordés, et si l'agrément validé devrait jouer un rôle.

Il reste encore beaucoup de travail à faire pour cerner les modèles optimaux d'exercice de la pharmacie en établissement de santé, y compris des études d'efficacité comparée pour déterminer les modèles particuliers qui permettent d'obtenir les meilleurs résultats concernant les soins aux patients. Néanmoins, les services de pharmacie des établissements de santé canadiens continuent d'innover en matière de modèles d'exercice, ont de quoi être très fiers et ont, sans l'ombre d'un doute, une longueur d'avance.

[Traduction par l'éditeur]

\section{Références}

1. American Society of Health-Systems Pharmacists. Pharmacy Practice Model Summit. Executive summary. Am J Health Syst Pharm 2011;68(12):1079-1085.

2. The consensus of the Pharmacy Practice Model Summit. Am J Health Syst Pharm 2011;68(12):1148-1152.

3. Zellmer WA. Reflections on the key messages of the Pharmacy Practice Model Summit. Am J Health Syst Pharm 2011;68(12):1153-1154.

4. Zellmer WA, Cobaugh DJ, Chen D. Three signals from the Pharmacy Practice Model Summit [éditorial]. Am J Health Syst Pharm 2011;68(12):1077.

5. Berry R. Cutting-edge practice model: experience in a small community hospital. Am J Health Syst Pharm 2011;68(12):1113.

6. Mason KC. Cutting-edge practice model: an integrated model within a large academic medical center. Am J Health Syst Pharm 2011;68(12):1112.

7. Sonnedecker G. Kremers and Urdang's history of pharmacy. $4^{\mathrm{e}}$ édition. Madison (WI) : American Institute of the History of Pharmacy; 1976. p. 241.

8. Francke DE. Prescription for pharmacy practice 1984. Drug Intell Clin Pharm 1976;10(2):111-112.

9. Hall KW, Raymond CB, Woloschuk DMM, Honcharik N. Organizational restructuring of regional pharmacy services to enable a new pharmacy practice model. Can J Hosp Pharm 2011;64(6):451-456.

10. Yuksel N, Eberhart G, Bungard TJ. Prescribing by pharmacists in Alberta. Am J Health Syst Pharm 2008;65(22):2126-2132.

James E Tisdale, B. Sc. Pharm., Pharm. D, est professeur titulaire et chef intérimaire du Département de pratique pharmaceutique de la Faculté de pharmacie de I'Université Purdue et professeur associé de l'École de médecine de I'Université d'Indiana, à Indianapolis, en Indiana, aux ÉtatsUnis. II est également rédacteur adjoint du JCPH.

Kevin W Hall, B. Sc. Pharm., Pharm. D., est professeur agrégé de clinique à la Faculté de pharmacie et des sciences pharmaceutiques de l'Université de l'Alberta, à Edmonton, en Alberta. Il est également rédacteur adjoint du JCPH

\section{Adresse de correspondance :}

D' James E Tisdale

Department of Pharmacy Practice

College of Pharmacy, Purdue University

W7555 Myers Building, WHS

1001 West 10th Street

Indianapolis IN 46202 USA

Courriel : jtisdale@purdue.edu

D' Kevin W Hall

Faculty of Pharmacy and Pharmaceutical Sciences

ECHA 3-228, University of Alberta

Edmonton AB T6G 1C9

Courriel : khall@pharmacy.ualberta.ca 